\title{
Three-Dimensional Ultrasonography, Virtual Coloscopy and Endorectal Magnetic Resonance Imaging in the Diagnosis of Complicated Inflammatory Bowel Disease
}

The main abdominal complications of Crohn's disease are the strictures, fistulas and abscesses [1]. Perianal disease occurs in up to $90 \%$ of patients with Crohn"s disease [2]. Endorectal ultrasonography and magnetic resonance imaging (MRI) are the most sensitive methods for detecting fistulas and abscesses in Crohn's disease [3]. The use of endoscopic ultrasonography (EUS) can change clinical management in a variety of anorectal conditions [4]. We report a case of complicated Crohn's disease and its diagnosis by means of these new methods.

A 43-year-old woman was admitted into the hospital because of her antalgic walk. She had experienced walking disorders for a year, but a rheumatological origin had been excluded before she was admitted to the hospital. Infection was excluded by means of polymerase chain reaction (PCR). Three-dimensional (3D) abdominal ultrasound showed a narrowing in the distal colon region, without lymph nodes. Endoscopic examination revealed a lumen in the same location which was endoscopically impenetrable. CT colonography was carried out after glucagon administration. The bowel was distended with air and spiral scans were performed. The CT colonography showed a narrowing of $3 \mathrm{~cm}$ in length in the distal part of the descending colon (Figure 1). Because of the patient's antalgic gait, rectal 3D EUS

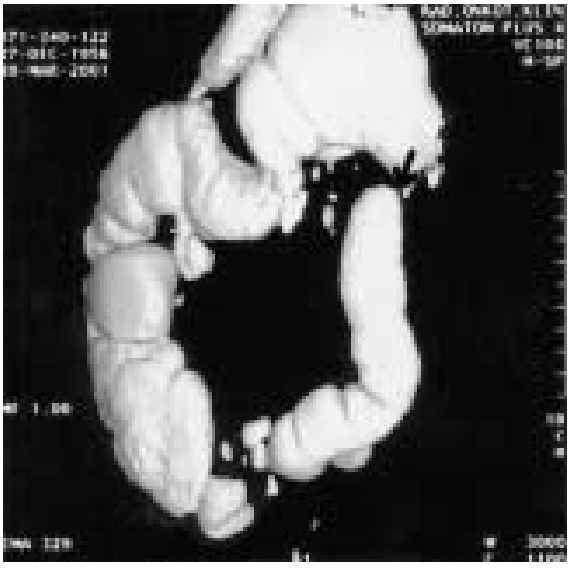

Figure 1 Computed tomography (CT) colonography showed a narrowing $3 \mathrm{~cm}$ in length (arrowed) at the distal part of the colon

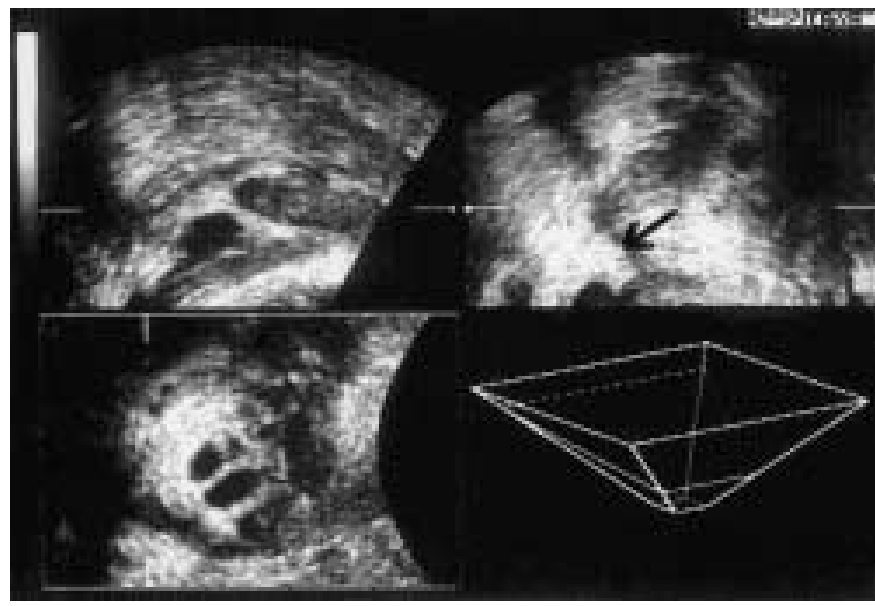

Figure 2 Threedimensional endoscopic ultrasonography (3D EUS) showing perirectal inhomogenic lesion and fistula. The fistula is arrowed

was performed, which revealed a perirectal inhomogenous lesion (Figure 2). The lesion was connected with an echo-poor narrowing (fistula). The fistula was detectable as far as the narrowed colon. Endorectal MRI examination at the axial FAST T $_{2}$, STIR, and sagittal FAST T 2 STIR native and postcontrast $T_{1}$ sequences were performed. An irregularly contoured region of $1.5 \mathrm{~cm}$ in diameter was detected next to the rectum, with a high accumulation of contrast agent and which was chracterized by inflammation. Genital and musculosceletal origins were excluded, and Crohn's disease was confirmed by surgical and histological examination.

The case presented here demonstrates the diagnostic problems of complicated inflammatory bowel disease. Investigative modalities for perianal Crohn's are changing because of the limitations of conventional evaluation [4].

\section{Á. Szilvás ${ }^{1}$, G. Székely ${ }^{2}$, Z. Tarján ${ }^{3}$, B. Fornet ${ }^{4}$}

${ }^{1}$ First Dept. of Internal Medicine and Gastroenterology, Bajcsy-Zsilinszky Hospital, Budapest, Hungary

${ }^{2}$ First Dept. of Internal Medicine and Gastroenterology, St. John's Hospital, Budapest, Hungary

${ }^{3}$ Radiological and Oncotherapeutic Clinic, Semmelweis University, Budapest, Hungary

${ }^{4}$ International Medical Center, MÁV Hospital, Budapest, Hungary
References

${ }^{1}$ Maconi G, Bollani S, Bianchi-Porro G. Ultrasonographic detection of intestinal complications in Crohn's disease. Dig Dis Sci 1996; 41(8): 1643-1648

${ }^{2}$ Solomon MJ. Fistulae and abscesses in symptomatic perianal Crohn's disease. Int J Colorectal Dis 1996; 11(5): 222 226

${ }^{3}$ Limberg B. Diagnosis of chronic inflammatory bowel disease by ultrasonography. Z Gastroenterol 1999; 37(6): 495 508

${ }^{4}$ Solomon MJ, McLeod RS, Cohen EK et al. Reliability and validity studies of endoluminal ultrasonography for anorectal disorders. Dis Colon Rectum 1994; 37(6): $546-551$

Corresponding Author

\section{Á. Szilvás}

I Dept. of Internal Medicine and Gastroenterology

Bajcsy-Zsilinszky Hospital

Maglódi str. 89-91

1106 Budapest

Hungary

Fax: + 36-1-261-9591

E-mail: bajcsy@matavnet.hu 